\title{
BMJ Open Educators' perspectives about how older hospital patients can engage in a falls prevention education programme: a qualitative process evaluation
}

\author{
Anne-Marie Hill, ${ }^{1,2}$ Steven M McPhail, ${ }^{3,4}$ Jacqueline Francis-Coad, ${ }^{1,2}$ \\ Nicholas Waldron, ${ }^{5,6}$ Christopher Etherton-Beer, ${ }^{7}$ Leon Flicker, ${ }^{7}$ Katharine Ingram, ${ }^{8}$ \\ Terry P Haines ${ }^{9,10}$
}

To cite: Hill A-M,

McPhail SM, Francis-Coad J, et al. Educators' perspectives about how older hospital patients can engage in a falls prevention education programme: a qualitative process evaluation. BMJ Open 2015;5:e009780. doi:10.1136/bmjopen-2015009780

- Prepublication history for this paper is available online. To view these files please visit the journal online (http://dx.doi.org/10.1136/ bmjopen-2015-009780).

Received 20 August 2015 Revised 18 October 2015 Accepted 13 November 2015

CrossMark

For numbered affiliations see end of article.

Correspondence to Dr Anne-Marie hill; anne-marie.hill@nd.edu.au

\section{ABSTRACT}

Objectives: Falls are the most frequent adverse event reported in hospitals. Patient and staff education delivered by trained educators significantly reduced falls and injurious falls in an older rehabilitation population. The purpose of the study was to explore the educators' perspectives of delivering the education and to conceptualise how the programme worked to prevent falls among older patients who received the education.

Design: A qualitative exploratory study.

Methods: Data were gathered from three sources: conducting a focus group and an interview $(n=10$ educators), written educator notes and reflective researcher field notes based on interactions with the educators during the primary study. The educators delivered the programme on eight rehabilitation wards for periods of between 10 and 40 weeks. They provided older patients with individualised education to engage in falls prevention and provided staff with education to support patient actions. Data were thematically analysed and presented using a conceptual framework.

Results: Falls prevention education led to mutual understanding between staff and patients which assisted patients to engage in falls prevention behaviours. Mutual understanding was derived from the following observations: the educators perceived that they could facilitate an effective three-way interaction between staff actions, patient actions and the ward environment which led to behaviour change on the wards. This included engaging with staff and patients, and assisting them to reconcile differing perspectives about falls prevention behaviours.

Conclusions: Individualised falls prevention education effectively provides patients who receive it with the capability and motivation to develop and undertake behavioural strategies that reduce their falls, if supported by staff and the ward environment.

\section{INTRODUCTION}

Older patients in rehabilitation wards are at increased risk of falls compared to other

\section{Strengths and limitations of this study}

- The participants in the study included all educators from the primary study and as such provided comprehensive feedback. These health professionals delivered the programme to over 750 patients across eight rehabilitation wards.

- Data were gathered from three sources which provided a robust and deep understanding of the educators' perspectives about how the education programme was able to facilitate patients to engage in falls prevention behaviours.

- As qualitative data, the findings may not be able to be directly translated to other settings where ward procedures regarding mobility and communication differ from the setting described.

hospital areas with reported rates of between 10 and 20 falls per 1000 patient days. ${ }^{1-3}$ Between $30 \%$ and $40 \%$ of in-hospital falls result in physical injury and approximately $2 \%$ result in hip fractures. ${ }^{4}$ Meta-analyses have found that multifactorial interventions can reduce falls in hospital settings but these interventions have included differing combinations of strategies that target the environment, staff or patients, meaning that specific recommendations for interventions are often uncertain. ${ }^{6}$

Previous studies that have investigated how to reduce falls in hospital have shown there is some evidence that patient education is of benefit when provided as a part of multifactorial programmes ${ }^{7-9}$ and as a single intervention. ${ }^{4} \mathrm{~A}$ recent meta-analysis of patient education interventions for reducing falls in hospital and after discharge found that providing individualised patient education increased patients' knowledge and awareness of falls prevention strategies. ${ }^{10}$ Qualitative studies conducted in hospital settings have 
also found that patient knowledge and communication to patients about falls prevention is an essential component of a comprehensive patient care plan to prevent falls. $^{11-14}$

Studies describing the circumstances of falls in hospital demonstrate that over $80 \%$ of falls occur when patients are unassisted, that is engaging in activities on the ward, such as toileting or moving from bed to chair, without direct supervision by staff. ${ }^{15} 16$ A recent large USA cross-sectional analysis of over 165000 falls found that excluding repeated falls, $85.5 \%$ of falls were unassisted, ${ }^{17}$ suggesting that patients may overestimate their functional abilities and require more education about how to safely complete required ward activities. Recently we conducted a large stepped-wedge cluster randomised controlled trial (RCT) ${ }^{18} 19$ which found that providing individualised falls prevention education to older hospital patients and providing training and feedback to staff to support the education in addition to usual care, significantly reduced falls and injurious falls. The full description of this intervention, the Safe Recovery program, is provided elsewhere. ${ }^{4} 1920$ The programme aimed to provide older patients education designed and delivered using health behaviour change principles $^{21} 22$ with awareness of the risk of falls in hospital, knowledge about falls and falls prevention strategies and the confidence and motivation to engage in planned falls prevention behaviours while in hospital. As part of the ward level intervention delivery, the educator also provided staff with education and training to allow them to support patients to engage in the desired falls prevention behaviours. ${ }^{19}$

Therefore the investigators considered the educators' insight to be important in understanding how the provision of individualised falls prevention education was translated into effective engagement in falls prevention behaviours. The purpose of the present study was to explore the educators' perspectives of delivering the Safe Recovery patient education program on rehabilitation units and to conceptualise their perception of how the programme worked to prevent falls among older patients who received the education.

\section{METHODS}

\section{Ethics}

This study was approved by The University of Notre Dame Australia and The Sir Charles Gairdner Group Human Research Ethics Committees. Numbers 2012_141 and 012069F. All 10 educators were invited to take part in the study and all provided written consent.

\section{Design}

A qualitative exploratory study which was part of a sequential mixed methods study. ${ }^{23}$ The qualitative approach used a focus group and interview, ${ }^{24}$ participant (educator) notes written at the end of the study and reflective researcher field notes based on interactions with the educators over a 12-month period. The study was conducted immediately following the quantitative study phase. ${ }^{18} 19$

\section{Trial intervention}

The Safe Recovery Program was a ward level in-hospital falls prevention education intervention, which included individualised education delivered directly to patients and staff. The intervention has also been described previously. ${ }^{19}{ }^{20}$ Briefly, patients who were eligible to directly receive education were 60 years or older and did not have moderate or severe cognitive impairment (mini mental state examination $>23 / 30$ ) ${ }^{25}$ The individualised education consisted of providing patients with a multimedia package (a DVD to view and a written workbook to read) followed up by between one and three individualised sessions with a physiotherapist (educator) trained to provide the education. The educators also provided education to multidisciplinary staff to facilitate their support of the programme as described previously. ${ }^{19}$ This was provided by conducting short presentations with groups of staff to explain the programme, with these repeated for new staff. Staff were also provided with weekly feedback on the ward formally and informally, ${ }^{20}$ about goals that patients were setting and patients' observations about the ward environment regarding safe mobility, for example, if patients noticed clutter on their wards.

\section{Participants and setting}

All physiotherapists $(\mathrm{N}=10)$ trained as 'Educators' in delivering the Safe Recovery program were invited to participate in the focus group. The programme was implemented in eight aged care rehabilitation hospital units in Western Australia. During the intervention phases of the trial the educators attended the wards two to three times per week. Eight educators were experienced in working with older people in hospitals, and two new graduate physiotherapists delivered the education programme for approximately 8 weeks at multiple sites when any of the educators were on leave. The educators were provided by the university research team and were not employed at the hospital. They were trained in understanding health behaviour change theory, ${ }^{21}{ }^{22}$ adult learning principles, ${ }^{26}$ the epidemiology of falls in hospital wards, facilitating older patients to develop and engage in individualised planned falls prevention strategies and engaging with staff to provide education about the programme and feedback about patient goals.

\section{Data gathering and procedure}

The focus group ran for $2 \mathrm{~h}$ and was conducted at the university. This was considered a neutral venue away from the hospital sites, where the educators would be able to discuss their reflections about the delivery of the programme in confidence. The researchers were experienced aged care physiotherapists who had received training in qualitative methods and had conducted 
focus groups previously. The focus group was moderated by the first researcher (AMH) and assisted by a second researcher (JFC) who took notes including description of key points and emphasis that could not be captured by audio recording alone. The moderator started the focus group with participant introductions, then presented the aims of the focus group and discussed how the information would be used. Participants were encouraged to discuss, rather than to find consensus, and to deeply explore the barriers and facilitators to delivery and uptake of the education messages.

The guiding questions for the focus group were developed by scrutinising the first researcher's reflective field notes which were collected during the trial. The field notes were compiled using de-identified information from email, short-message service (text message) and informal phone discussions conducted between the researcher and all 10 educators during the period when the Safe Recovery program was being delivered. The educators emailed and sent text messages to the researcher with observations and questions regarding delivery of the Safe Recovery program, including any problems noted. Educators were also encouraged to participate in group email and text message discussion with each other to share information. There were approximately 40 separate emails and more than 50 primary text messages between the researcher and the educators, which generated multiple correspondences through ongoing discussion threads, phone messages and phone communication. The two researchers independently read these field notes to determine a series of broad guiding questions. These were compared and consensus reached. These two researchers developed draft guiding questions, then sought input from a third researcher (SMM) who had previously delivered the Safe Recovery program in other hospitals, but had not been involved in delivery or data collection during this trial. The guiding questions that were used to structure the focus group discussion were:

1. What are the facilitators and barriers to the delivery of the Safe Recovery program?

2. What are the facilitators and barriers that influence patients' engagement in their chosen falls prevention strategies?

3. What feedback did the educator receive from patients and from staff on the wards about the delivery of the Safe Recovery program?

Prior to the focus group the educators were asked to reflect on their own perspective and to write down their thoughts and responses to the guiding questions. At the close of the focus group the participants were reminded to write down any additional comments that they perceived were relevant to exploring their delivery of the Safe Recovery program. It was emphasised that these notes would also be analysed confidentially and as augmentation with the focus group transcript. These written notes, which ranged between two and four pages in length, were collected after the session concluded.

\section{Analysis}

Two researchers (AMH and JFC) independently listened to the audio recording from the focus group; the first researcher $(\mathrm{AMH})$ transcribed the audio data verbatim and the second researcher (JFC) then read the transcript checking for accuracy against the audio recording. The researchers also read and familiarised themselves with participants' notes and the field notes that had been transcribed. Qualitative data obtained from these three data sources were analysed using thematic analysis. ${ }^{27}$ Data familiarisation is a key to thematic analysis and in this study the researchers personally collected and transcribed their data themselves.

Data from all three sources (researcher field notes, focus group and interview and participant (educator) notes) were used with the intent to triangulate the different sources of data to gain a multilayered understanding of the findings. ${ }^{27}$ These data were separated into individual response items and managed using NVivo Version10 (NVivo qualitative data analysis software; QSR International Pty Ltd. V.10, 2012). The first researcher examined the data and coded each item, with open coding used: several iterations of grouping and regrouping took place to fit all items into identified codes. These coded data were then examined to identify candidate themes. Each candidate theme was then re-examined to ascertain if it described the data collected and if all coded data were captured within these identified candidate themes. Quotes illustrating each theme were identified for use when presenting the final results. The second researcher examined the coded data independently and also identified candidate themes from the coded data. These two researchers then compared and discussed the coding and resultant themes, moving back and forth within the data to ascertain that themes captured the meaning of the coded data, and revising where necessary the fit of the coded data into each theme. The third researcher (SMM) who was not involved in data collection or site supervision, was then invited to scrutinise the data and to arbitrate any differences between coding and themes. This method of analysis provided researcher triangulation which aimed to obtain a broader picture of the data. ${ }^{27}$ The coded data and themes were then reviewed to identify any initial overarching themes and an initial framework ${ }^{28}$ was constructed, which aimed to capture the relationship between the initial themes and coded data. The framework was then examined to see if it adequately explained the relationship of these overarching themes to themes and coded data, and captured the overall data set. This assisted all three researchers to achieve consensus in confirming the final themes and constructing the final conceptual framework. This framework assisted to explain the educators' perspective about how the education programme worked to prevent falls on the hospital wards. Member checking was used to seek feedback and clarification of the results with the themes and conceptual framework sent to the participants. Any feedback 
was noted and differences arising were resolved by consensus from all three researchers. ${ }^{27}$

\section{RESULTS}

The researchers conducted a single focus group $(n=9)$, from the total population of 10 educators who delivered the Safe Recovery program. Nine of the 10 educators (P1-P9) who delivered the education participated in the focus group. The remaining educator, who was unable to attend the focus group session, was followed up with a telephone interview using the same questions administered in the focus group. At this point all data available to answer the research question had been collected. ${ }^{24}$ The new two new graduate educators $(\mathrm{P} 8, \mathrm{P} 10)$ who participated in the focus group worked for 8 weeks and provided the programme at three hospitals. The other educators provided the programme for between 10 (P3, P4) and 40 weeks $(\mathrm{P} 1, \mathrm{P} 2)$ at least one hospital. New graduate perspectives did not differ from the other educators.

Coded data were initially grouped into three emerging subthemes which were named according to their collective meaning. These subthemes reflected the underlying elements of the education programme: the delivery of the education to patients ('patient education'), delivery of supportive education to staff ('staff education') and the ward environment ('the ward environment impacting on the delivery of the education').

\section{Patient education}

The educators reported that patients needed to be exposed to the education programme in a manner that highlighted its personal applicability such as by stating 'we're working towards you becoming more independent like you were before' (P1). Educators reported that patients required specific instruction and practice in specific actions such as "waiting for staff before going to the toilet,' which meant that they tailored the programme for each patient. Patients also needed to understand the rehabilitation process with educators suggesting they 'talk about the journey to independence is (in) safe stages' (P8), or they could be reluctant to accept assistance or be motivated to engage in falls prevention behaviours. Educators observed that many patients felt empowered to monitor their own safety after receiving the Safe Recovery program.

So it empowers the patient makes them feel more confident that they can express themselves to the nursing staff...for example saying by the way would you mind putting that bell back in reach before you go. (P6).

\section{Staff education}

Exposure through education and subsequent acceptance and 'promotion of the program' (P6) by nursing and other staff was viewed as an essential element to support effective delivery of the education programme.
Educators provided group and individual information and training sessions for staff, but perceived that staff response was more supportive when the purpose of the education programme was explained using real clinical examples. This meant that informal education often took place at patients' bedsides and was discussed as relevant for that patient with educators commenting that " the best is to get them on the job with their patient, explain what you do, chat about that patient' (P2).

We all have the same goal in regards to the patients and I think it helps to tell the nurse that sometimes-(give) education that this can reduce their workload in the long run. (P4).

\section{Ward environment impacting on delivery of the education} Environmental factors on the ward were viewed by the educators as being controlled by staff and also to some extent by patients, and could be supportive or inhibitory to patients being able to effectively enact planned behaviours such as ringing their bell for assistance. Communication about mobility was strongly felt to be a key environmental factor. In these rehabilitation wards, the level of assistance that patients required with mobility tasks was documented in a chart which was kept at the bedside, with further information available in the care plan. Educators felt that the patient's mobility status needed to be understood and agreed on by all staff. Patients' use of the bell was also viewed as critical to mobilising safely because nearly all patients in the rehabilitation setting required assistance or supervision to mobilise, while undergoing rehabilitation. Therefore educators considered it essential that the bell was consistently placed within reach by staff and patients and some educators noted that 'bell accessibility can be a challenge' (P1).Observations about bell use applied to other mobility items, such as walking aids, splints and appliances, glasses and shoes. The most common problem was leaving the appliance or aid 'out of reach' (P9).

\section{Facilitators and barriers to effective delivery of the education}

These subthemes, 'patient and staff education and the ward environment' impacting on the delivery of the education were able to be grouped into two larger themesthese were 'Facilitators' of effective delivery and 'Barriers' to effective delivery of the Safe Recovery education program. The educators identified that there were facilitators that encouraged and supported patients to take up safe falls prevention behaviours, whereas barriers prevented or mitigated against patients engaging in their planned behavioural goals.

The educators concurred that the education acted as a facilitator to nearly all patients to take up falls prevention behaviours as they saw the personal application and 'believed that the strategies are helpful, a realisation that 
this does apply to them' (P9 notes). However, they also noted that patients could be reluctant to take action and could require encouragement to enact their planned goals.

Making patients feel firm to say you cannot leave the room, I need my bell, I need my telephone, and that was quite difficult because patients were quite afraid to make a comment. (P2)

All educators interacted with a small group of patients who incorrectly viewed themselves as not being at risk of falls and perceived that they were able to maintain their independence regardless of their diagnosis with one commenting that 'there is a certain type of patient it's often a younger male, that is resistive to the education' (field notes). These beliefs were perceived as a barrier by the educators. However, educators noted that most of these patients were still willing to set goals and write an action plan to engage in safe mobility. Educators also perceived that it was difficult to empower or facilitate patients to enact their goals if patients perceived that individual staff members were not supportive of their goals. One educator reported that 'hostile nursing staff when patient seeks assistance lowers patient engagement' (field notes). Conversely educators noted that staff being open to change, such as 'cooperative friendly staff who re-enforce the message' (P1), facilitated patient engagement.

Regarding the environment, placement of aids and the bell could facilitate engagement in safe strategies if done correctly by staff and patients such as 'keep walking aid near especially if patient is independent, so they don't feel tempted to get it themselves' (P2). Regarding the mobility charts, educators felt that the patient's mobility status needed to be understood and agreed upon by all staff or 'patients can be confused about their mobility and level of independence' (P9). Even when charts were accurate it was also seen that this could still be a barrier to engagement in safe mobility, if either patients or staff did not follow the chart recommendations.

You educate the patient who has been assessed by staff on their mobility chart as requiring assistance, about being safe and ask for assistance then a nurse will go in and say you need to be independent so it's a total contradiction. (P9).

\section{Overarching themes}

When these subthemes and themes were further reviewed within the context of the research question, two overarching themes were identified. These were 'engagement' and 'reconciliation.

\section{Engagement}

The educators emphasised that a key factor for successful delivery was one of building engagement between themselves, patients and staff, which subsequently facilitated uptake of key education messages by each group. They were strongly convinced that the education process was only successful if they gained an effective level of engagement from staff 'having staff on board' (P3), and patients 'you need a good sell so that patients identify with your delivery' (P5). The education programme was strongly collaborative in nature, with patients being viewed as adult learners and the educators perceived that patient engagement was critical to ensure its success. However, it was a ward level intervention and the programme was facilitated by the educator gaining effective engagement with staff.

\begin{abstract}
So much of it comes to the rapport so you are the person who sits down for a chat and to look them in the eye... and patients are really happy, they look to you. (P4)
\end{abstract}

\section{Reconciliation}

As an independent mediator, who understood a hospital ward environment, educators perceived that they were able to assist to reconcile differences that emerged between patients and staff, regarding set up of the ward environment and how patients should complete mobility tasks in a manner that reduced their risk of falls: 'patients often say staff move all my things around and don't come back so that is the feedback I am often giving to staff' (P6). Educators were able to provide independent feedback from patients to staff as 'an independent person on the ward (who) allows patients to express their views and thoughts' (P8). As part of this reconciliatory process, they viewed their role as requiring them to advocate for patients to staff to assist in reconciling staff instructions with patients' and other staffs' understanding.

Then after I saw the patient I might go back to the physio and say could you write something more specific about night time (mobility) just to try to make it less open to interpretation. (P1)

The final conceptual framework which answered the research question of how the educators conceptualised the programme worked to prevent falls by older patients is presented in figure 1 . The educators engaged with staff and patients, and facilitated reconciliation of their understanding about falls prevention, patient mobility and the ward environment. This led to a mutual understanding between staff and patients about the actions required within the ward environment to prevent falls from occurring. Behaviour change in patients was conceptualised as occurring through an effective or ineffective three-way interaction between staff, patients and the environment and was mediated by the Safe Recovery education. The framework elements were consistent with the theory of health behaviour change, where knowledge, motivation and opportunity are required for behaviour change to occur. ${ }^{21}{ }^{22}$ Patients developed knowledge and awareness about falls prevention, and motivation to engage in falls prevention behaviours. 
Misunderstanding between the patient and staff is likely to result in a fall

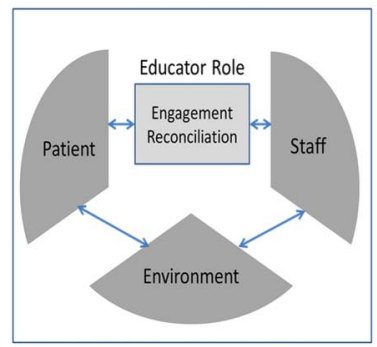

Mutual understanding between the patient and staff is likely to prevent a fall

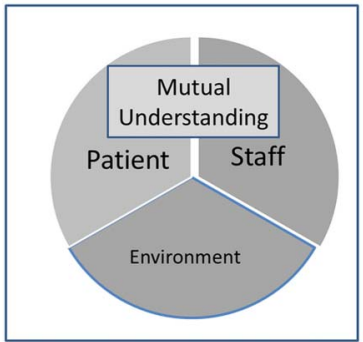

Figure 1 Conceptual framework for falls prevention -Mutual understanding between patient and staff facilitates effective falls prevention behaviours.

Social opportunity (physical and psychological) was provided by staff and patients modifying the environment and by staff providing support for patients when they engaged in their action plan. Engagement in falls prevention behaviours was less likely to occur when there were misunderstandings between patients and staff about the behaviours required on the ward. Engagement in falls prevention behaviours was more likely to occur when patients and staff arrived at a mutual understanding about the patient's safe level of function and mobility. Two examples are presented (table 1) which illustrate how the educators reported that they engaged patients and staff and facilitated reconciliation of differing perspectives to prevent falls by older inpatients. This led to mutual understanding, which resulted in patients engaging in effective falls prevention behaviours on the wards.

\section{DISCUSSION}

This study explored how an effective falls prevention education programme worked in a rehabilitation hospital ward from the perspective of the educators. The conceptual framework explained educators' perspective that all three domains (patient, staff and environment) must interact effectively to facilitate engagement in falls prevention behaviours. In addition to engaging staff and patients to facilitate knowledge gain, the educators perceived that a key aspect of their role was to reconcile patients and staff understanding about how patients could engage in planned safe behaviours in a supportive ward environment. The educators believed that feedback they provided to staff was crucial in developing a mutual understanding which empowered patients to engage in safe behaviours, such as ringing the call bell for assistance. This was important because when this intervention was previously delivered to patients without staff support, falls reduction only occurred in a subgroup of patients and not across the whole ward. ${ }^{4}$ Other studies have also identified that understanding between staff and patients, combined with environmental safety, including ringing the call bell is important to reduce falls risk. ${ }^{29} 30$

Previous studies have identified that factors relating to the ward environment, ${ }^{31}{ }^{32}$ staff actions and training ${ }^{14} 33$ and patient intrinsic risk factors and actions ${ }^{34}$ contribute to the occurrence of falls in hospital wards. Large randomised trials have delivered interventions that have directed multifactorial interventions towards some or all of these three domains. ${ }^{6}$ However, the educators in our trial concurred that effective interaction of all three domains facilitated effective falls prevention behaviours on the ward. Hence engaging patient and staff, to reconcile their perspectives about the functional mobility that patients should undertake, facilitated an effective interaction of all three domains. This reconciliation is particularly important for patients who wish to test their physical independence as this has been shown to be linked to risk taking behaviour on inpatient wards. ${ }^{12}$

Health behaviour change theory conceptualises that older patients need to gain the capability and motivation for behaviour change. This is achieved by developing an accurate self-perceived risk of falls, knowledge about how to engage in suitable strategies and the ability to identify cues which alert them to engage in these falls

Table 1 Examples of effective three-way interactions between patients, staff and environment which promoted engagement in falls prevention behaviours

\begin{tabular}{|c|c|c|c|}
\hline Educator role & Patient & Staff & $\begin{array}{l}\text { Environment (mediated by } \\
\text { staff and patient) }\end{array}$ \\
\hline $\begin{array}{l}\text { Patient individually educated on } \\
\text { reasons why and how to take relevant } \\
\text { action of ringing bell: educator } \\
\text { advocates to staff about building } \\
\text { patient confidence to ring bell }\end{array}$ & $\begin{array}{l}\text { Avoids risk taking } \\
\text { behaviour-rings bell } \\
\text { for assistance }\end{array}$ & $\begin{array}{l}\text { Patient encouraged to ring } \\
\text { bell: bell answered in } \\
\text { timely manner }\end{array}$ & $\begin{array}{l}\text { Bell working and in reach at all } \\
\text { times }\end{array}$ \\
\hline $\begin{array}{l}\text { Patient individually educated on } \\
\text { reasons why and how to take relevant } \\
\text { action of using mobility aid: educator } \\
\text { clarifies with both staff and patient to } \\
\text { eliminate differing perceptions of } \\
\text { mobility levels }\end{array}$ & $\begin{array}{l}\text { Avoids risk taking } \\
\text { behaviour-uses } \\
\text { mobility aid correctly }\end{array}$ & $\begin{array}{l}\text { Staff consistent on their } \\
\text { instruction about use of } \\
\text { mobility aid and the level } \\
\text { of assistance they provide } \\
\text { to patients }\end{array}$ & $\begin{array}{l}\text { Mobility aid available, correctly } \\
\text { prescribed and in reach at all } \\
\text { times }\end{array}$ \\
\hline
\end{tabular}


prevention strategies. ${ }^{21} 2235$ However, the final component of behaviour change is opportunity to enact planned goals, ${ }^{22}$ which in this context equates to falls prevention behaviours. Therefore ward staff have an important role in this process through their influence on the environment and by providing opportunities for facilitating patient engagement in suitable preventive behaviours. The educators reported that a high degree of mutual understanding between patients and staff was required for falls prevention behaviours to be enacted. In this regard, educators felt they could act as advocates for patients to assist to reconcile the views of staff and patients. These findings when viewed from a health behaviour context assist to explain why the education programme was effective. The educators felt that the programme provided staff on the ward with information about patient perspectives, which was particularly effective when informally provided. Educators could feedback to staff if they found that patients felt that messages were inconsistent or confusing and further, they could empower patients to actively seek out accurate information about their falls risk and mobility status.

A key strength of this study was that data were obtained from the educators that delivered the Safe Recovery program in a large, rigorous RCT that significantly reduced the number of falls, fallers and injurious fall events. ${ }^{18}$ The researchers asked the educators to reflect on what they perceived made the Safe Recovery program effective as they interacted with patients and staff. This is important to consider when interpreting these qualitative findings regarding the educatorperceived mechanisms of effect from this investigation, on account that the falls prevention effect was not theoretical or hypothesised, but real and observable. The perspectives of the entire population of educators, who delivered education to over 750 patients across eight wards, were captured. Patients with impaired cognition did not directly receive education, therefore investigating the perspective of ward staff who interacted with patients with impaired cognition remains an important avenue for further research. The interpretation of data reported in this investigation was potentially influenced by the lead researcher's perspective as the physiotherapist who conducted the larger trial. To counter this potential bias the second focus group researcher was a physiotherapist who did not assist to conduct the trial and the analysis was completed by three researchers, two of whom were not involved in data collection or trial procedure, with convergent findings.

The educators provided effective patient education across eight hospitals with different organisational cultures and heterogeneous staff and patient populations. Therefore although qualitative data have limited generalisability, it is likely these findings are robust for the purpose of understanding how this falls education programme influenced falls risk taking behaviours and ultimately reduced the rate of falls (and injuries), as well as factors that may potentially facilitate or inhibit desired behaviour change by patients. It is particularly important for understanding how educators can effectively deliver falls prevention education on inpatient wards. Future research should also seek to understand this successful education programme from patient and staff perspectives, which will provide further understanding of how effective falls prevention education can be delivered on hospital wards.

\section{Author affiliations}

${ }^{1}$ School of Physiotherapy, The University of Notre Dame Australia, Fremantle, Western Australia, Australia

${ }^{2}$ Institute for Health Research, The University of Notre Dame Australia, Fremantle, Western Australia, Australia

${ }^{3}$ Centre for Functioning and Health Research, Metro South Health, Brisbane, Queensland, Australia

${ }^{4}$ Institute of Health and Biomedical Innovation and School of Public Health \& Social Work, Queensland University of Technology, Brisbane, Queensland, Australia

${ }^{5}$ Department of Rehabilitation and Aged Care, Armadale Kelmscott Memorial Hospital, Perth, Western Australia, Australia

${ }^{6}$ Department of Health Strategy and Networks, Strategic System, Policy \&

Planning, Department of Health, Government of Western Australia, Perth, Western Australia, Australia

${ }^{7}$ WA Centre for Health and Ageing, Royal Perth Hospital Unit, School of Medicine \& Pharmacology and Centre for Medical Research, University of Western Australia, Perth, Western Australia, Australia

${ }^{8}$ Department of Rehabilitation, Aged Care Sir Charles Gairdner Hospital, Perth, Western Australia, Australia

${ }^{9}$ Physiotherapy Department, Monash University, Melbourne, Victoria, Australia

${ }^{10}$ Allied Health Research Unit, Monash Health, Melbourne, Victoria, Australia

Acknowledgements AMH is supported by a National Health and Medical Research Council (of Australia) early career fellowship. SmMcP and TPH are supported by National Health and Medical Research Council (of Australia) Career Development awards.

Contributors AMH contributed to study conception, design, intervention training, data collection and analyses, principal manuscript drafting and editing. SMMcP contributed to study conception, design, data management and analyses, manuscript drafting, appraisal and editing. JF-C contributed to study conception, design, data collection and analyses, manuscript drafting, appraisal and editing. NW, CE-B, LF and KI contributed to study conception, design, site management and provided advice on data collection and management. TPH contributed to study conception and design and intervention training. All authors contributed to manuscript appraisal, revision and editing and read and approved the final manuscript.

Funding This work was supported by the Western Australian State Health Research Advisory Council and the Department of Health, Western Australia as part of the Research Translation Projects program. This program was established to encourage research and translation of outcomes into health care policy and practice. The key aim of the projects is to demonstrate improved cost effectiveness and/or efficiencies to WA Health while maintaining or improving patient outcomes.

Competing interests None declared.

Ethics approval 1. The University of Notre Dame Australia HREC 2. North Metropolitan and South Metropolitan HRECS.

Provenance and peer review Not commissioned; externally peer reviewed.

Data sharing statement No additional data are available.

Open Access This is an Open Access article distributed in accordance with the Creative Commons Attribution Non Commercial (CC BY-NC 4.0) license, which permits others to distribute, remix, adapt, build upon this work noncommercially, and license their derivative works on different terms, provided the original work is properly cited and the use is non-commercial. See: http:// creativecommons.org/licenses/by-nc/4.0/ 


\section{REFERENCES}

1. Haines TP, Kuys SS, Morrison G, et al. Balance impairment not predictive of falls in geriatric rehabilitation wards. J Gerontol A Biol Sci Med Sci 2008;63:523-8.

2. Healey F, Monro A, Cockram A, et al. Using targeted risk factor reduction to prevent falls in older in-patients: a randomised controlled trial. Age Ageing 2004;33:390-5.

3. Schwendimann R, Bühler H, De Geest S, et al. Characteristics of hospital inpatient falls across clinical departments. Gerontology 2008;54:342-8.

4. Haines TP, Hill AM, Hill KD, et al. Patient education to prevent falls among older hospital inpatients: a randomized controlled trial. Arch Intern Med 2011;171:516-24.

5. Healey F, Scobie S, Oliver D, et al. Falls in English and Welsh hospitals: a national observational study based on retrospective analysis of 12 months of patient safety incident reports. Qual Saf Health Care 2008;17:424-30.

6. Cameron ID, Gillespie LD, Robertson MC, et al. Interventions for preventing falls in older people in care facilities and hospitals. Cochrane Database Syst Rev 2012;(12):CD005465.

7. Ang E, Mordiffi SZ, Wong HB. Evaluating the use of a targeted multiple intervention strategy in reducing patient falls in an acute care hospital: a randomized controlled trial. J Adv Nurs 2011;67:1984-92

8. Dykes PC, Carroll DL, Hurley AC, et al. Fall prevention in acute care hospitals: a randomized trial. JAMA 2010;304:1912-18.

9. Haines TP, Hill KD, Bennell KL, et al. Patient education to prevent falls in subacute care. Clin Rehabil 2006;20:970-9.

10. Lee DCA, Pritchard E, McDermott F, et al. Falls prevention education for older adults during and after hospitalization: a systematic review and meta-analysis. Health Educ J 2014;73:530-44.

11. Dykes PC, Carroll DL, Hurley AC, et al. Why do patients in acute care hospitals fall? Can falls be prevented? J Nurs Admin 2009;39:299-304.

12. Haines TP, Lee DC, O'Connell B, et al. Why do hospitalized older adults take risks that may lead to falls?. Health Expect 2015;18:233-49.

13. Lee DC, McDermott F, Hoffmann T, et al. 'They will tell me if there is a problem': limited discussion between health professionals, older adults and their caregivers on falls prevention during and after hospitalization. Health Educ Res 2013;28:1051-66.

14. Carroll DL, Dykes PC, Hurley AC. Patients' perspectives of falling while in an acute care hospital and suggestions for prevention. Appl Nurs Res 2010;23:238-41.

15. Hill AM, Hoffmann T, Hill K, et al. Measuring falls events in acute hospitals-a comparison of three reporting methods to identify missing data in the hospital reporting system. J Am Geriatr Soc 2010;58:1347-52

16. Hitcho EB, Krauss MJ, Birge S, et al. Characteristics and circumstances of falls in a hospital setting: a prospective analysis. J Gen Intern Med 2004;19:732-9.

17. Staggs VS, Mion LC, Shorr RI. Assisted and unassisted falls: different events, different outcomes, different implications for quality of hospital care. Jt Comm J Qual Patient Saf 2014;40: 358-64.

18. Hill AM, McPhail SM, Waldron N, et al. Reducing falls in rehabilitation hospital units using individualised patient and staff education: a pragmatic stepped-wedge cluster randomised controlled trial. Lancet 2015;385:2592-9.

19. Hill AM, Waldron N, Etherton-Beer C, et al. A stepped-wedge cluster randomised controlled trial for evaluating rates of falls among inpatients in aged care rehabilitation units receiving tailored multimedia education in addition to usual care: a trial protocol. BMJ Open 2014;4:e004195.

20. Hill AM, McPhail S, Hoffmann $\mathrm{T}$, et al. A randomized trial comparing digital video disc with written delivery of falls prevention education for older patients in hospital. J Am Geriatr Soc 2009;57:1458-63.

21. Janz NK, Becker MH. The Health Belief Model: a decade later. Health Educ Q 1984;11:1-47.

22. Michie S, van Stralen MM, West R. The behaviour change wheel: a new method for characterising and designing behaviour change interventions. Implement Sci 2011;6:42.

23. Cresswell JW. Research design: qualitative, quantitative and mixed methods approaches. London: Sage Publications, 2014.

24. Liamputtong P. Focus group methodology: principles and practice. Singapore: Sage, 2011.

25. Folstein MF, Folstein SE, Mchugh PR. "Mini-mental state." A practical method for grading the cognitive state of patients for the clinician. J Psychiatr Res 1975;12:189-98.

26. Swanson RA, Holton EF, Knowles MS. The adult learner: the definitive classic in adult education and human resource development. 7th edn, New York: Routledge, 2012.

27. Braun V, Clark V. Successful qualitative research; a practical guide for beginners. London: Sage, 2014.

28. Miles MB, Huberman AM, Saldana J. Qualitative data analysis-A methods sourcebook. London: Sage Publications, 2014.

29. Tzeng HM, Yin CY. Nurses' solutions to prevent inpatient falls in hospital patient rooms. Nurs Econ 2008;26:179-87.

30. Tzeng HM, Yin CY. Perspectives of recently discharged patients on hospital fall-prevention programs. J Nurs Care Qual 2009;24:42-9.

31. Grenier-Sennelier C, Lombard I, Jeny-Loeper C, et al. Designing adverse event prevention programs using quality management methods: the case of falls in hospital. Int J Qual Health Care 2002;14:419-26.

32. Tzeng HM. Perspectives of staff nurses of the reasons for and the nature of patient-initiated call lights: an exploratory survey study in four USA hospitals. BMC Health Serv Res 2010;10:52.

33. Australian Commission on Safety and Quality in Health Care. Guidebook for preventing falls and harm from falls in older people: Australian hospitals 2009[Online]. 2010. http://www.safetyandquality. gov.au/internet/safety/publishing.nsf/Content/com-pubs_ FallsGuidebook

34. Oliver D, Healey F, Haines TP. Preventing falls and fall-related injuries in hospitals. Clin Geriatr Med 2010;26:645-92.

35. Abraham $\mathrm{C}$, Michie S. A taxonomy of behavior change techniques used in interventions. Health Psychol 2008;27:379-87. 